\title{
MODULES AND RINGS SATISFYING (ACCR)
}

\author{
CHIN-PI LU
}

(Communicated by Louis J. Ratliff, Jr.)

\begin{abstract}
A module $M$ over a ring $R$ is said to satisfy (accr) if the ascending chain of residuals of the form $N: B \subseteq N: B^{2} \subseteq N: B^{3} \subseteq \cdots$ terminates for every submodule $N$ and every finitely generated ideal $B$ of $R$. A ring satisfies (accr) if it does as a module over itself. This class of rings and modules satisfies various properties of Noetherian rings and modules. For each of the following rings, we investigate a necessary and sufficient condition for the ring to satisfy (accr): polynomial rings, power series rings, valuation rings, and Prüfer domains. We also prove that if $R$ is a ring satisfying (accr), then every finitely generated $R$-module satisfies (accr).
\end{abstract}

\section{INTRODUCTION}

A module $M$ over a ring $R$ is said to satisfy (accr) if the ascending chain of residuals of the form $N: B \subseteq N: B^{2} \subseteq N: B^{3} \subseteq \cdots$ terminates for every submodule $N$ of $M$ and every finitely generated ideal $B$ of $R$. A ring satisfies (accr) if it does as a module over itself. It is known that an $R$-module $M$ satisfies (accr) if and only if the ascending chain of submodules of the form $N: b \subseteq N: b^{2} \subseteq N: b^{3} \subseteq \cdots$ terminates for every submodule $N$ of $M$ and every element $b$ of $R$ [11, Corollary to Theorem 1, p. 306]. The class of rings and modules satisfying (accr) is very large. It contains Noetherian modules, modules over Artinian rings, and more generally, modules satisfying the ACC on colon submodules [8], Laskerian modules, modules over perfect rings, zero-dimensional rings, one-dimensional domains, etc. Rings and modules satisfying (accr) have been investigated in [11]. One of the most interesting facts about this class of rings and modules is that they enjoy various properties of Noetherian rings or modules. In particular, coherent rings satisfying (accr) behave like Zariski rings if they are equipped with certain ideal-adic topologies [11, Proposition 8, Theorem 8, Corollary to Theorem 9].

The purpose of this paper is to explore more rings and modules satisfying (accr), in particular, non-Noetherian coherent rings satisfying (accr). Firstly, for each of the following rings, we investigate a necessary and sufficient condition

Received by the editors October 2, 1990 and, in revised form, April 5, 1991 and May 6, 1991; presented to the algebra session at the International Congress of Mathematicians (ICM), in Kyoto, Japan, on August 24, 1990.

1991 Mathematics Subject Classification. Primary 13E99, 13F05.

Key words and phrases. (accr), Laskerian ring, valuation ring, Prüfer domain. 
in order that the ring satisfies (accr): polynomial rings, power series rings, valuation rings, and Prüfer domains. Secondly, we study finitely generated modules over rings satisfying (accr).

In $\S 1$ we discuss that a polynomial ring $R[x]$ or a power series ring $R[[x]]$ satisfies (accr) if and only if $R$ is Noetherian (Theorem 2). In $\S 2$ we prove that a valuation ring satisfies (accr) if and only if $\bigcap_{n=1}^{\infty}\left(b^{n}\right)=(0)$ for every nonunit $b$ of the ring (Theorem 3). This implies a series of corollaries. For example, we have that a Prüfer domain $D$ satisfies (accr) if and only if $D$ has (Krull) dimension at most 1 . Thus, every almost Dedekind domain satisfies (accr). It follows that every Prüfer domain of dimension one is a coherent ring satisfying (accr) that is not necessarily Noetherian. The question asking whether every finitely generated $R$-module satisfies (accr) if $R$ satisfies (accr) has not been answered yet. In $\S 3$ we finally prove that the answer to the question is affirmative.

Every ring in this paper is commutative with identity and every module is unitary. For definitions not given in the paper the reader may refer to [11].

\section{FURTHER PROPERTIES OF MODULES AND RINGS THAT SATISFY (ACCR)}

In [11] various properties of modules and rings that satisfy (accr) were discussed. In this section, we investigate further properties of such modules and rings. We are also concerned about relationships between rings satisfying (accr) and other Noetherian-like rings.

Let $F$ be a submodule of an $R$-module $M$ and $T$ the set of all those submodules $K$ of $M$ that properly contain $F$. We say that $F$ is sheltered if the intersection $\bigcap_{K \in T} K$ also properly contains $F$ ([13, p. 27] or [5, Exercise 18 , p. 110]).

Proposition. Let $M$ be an $R$-module satisfying (accr). Then every proper submodule $N$ of $M$ has a (finite or infinite) primary decomposition $N=\bigcap_{i \in I} Q_{i}$, where each $Q_{i}$ is a primary submodule.

Proof. It is known that every proper submodule $N$ of any $R$-module is an intersection $\bigcap_{i \in I} Q_{i}$ of a family of sheltered submodules $Q_{i}$ ([13, Lemma 4.2, p. 27] or [5, Exercise 18, p. 110]). Since every sheltered submodule of a module is irreducible and every irreducible submodule of a module satisfying (accr) is primary by [11, Proposition 5, p. 307], $N=\bigcap_{i \in I} Q_{i}$ is a primary decomposition of $N$.

Let $I$ be an ideal of a ring $R$ and $\operatorname{comp}(I)$ be the set of prime ideals of $R$ that are minimal over $I . \quad R$ is said to have FC if $\operatorname{comp}(I)$ is finite for every ideal $I$ of $R$. Next, let $S(I)$ denotes the set complement of $U\{p: p \in \operatorname{comp}(I)\}$ in $R$. Then $I$ is said to have finite ideal-length if the ring $R_{S(I)} / I R_{S(I)}$ is an Artinian ring. $R$ is said to have finite ideal-lengths if each ideal of $R$ has finite ideal-length. It was shown in [3, Proposition 1.3, p. 2683] that $R$ has finite ideal-lengths if and only if $R$ has FC and satisfies the ACC for $p$-primary ideals for each prime ideal $p$ of $R$. Following Beachy and Weakley [3], we say that $R$ is piecewise Noetherian if it has finite ideal-lengths and has the ACC on prime ideals. Clearly, every Noetherian ring is piecewise Noetherian and every piecewise Noetherian ring has Noetherian spectrum. 
Theorem 1. Let $R$ be a ring such that each nonzero element of $R$ belongs to only finitely many maximal ideals of $R$. Then $R$ is Noetherian if and only if $R$ is a piecewise Noetherian ring satisfying (accr).

Proof. Suppose that $R$ is a piecewise Noetherian ring satisfying (accr). Then every maximal ideal of $R$ is finitely generated by [3, Proposition 2.2, p. 2689], and so $R$ is locally Noetherian due to [11, Corollary to Theorem 4, p. 312]. Now we can conclude that $R$ is Noetherian [2, Exercise 9, p. 85].

A ring $R$ is called a ZD-ring if the set of zero divisors on the $R$-module $R / A$ is a finite union of prime ideals for each ideal $A$ of $R$. Clearly, every Laskerian ring is a ZD-ring. Heinzer and Ohm [9] proved that a polynomial ring $R[x]$ is a ZD-ring if and only if $R$ is Noetherian, and hence the conditions Noetherian, Strongly Laskerian, Laskerian, and ZD are equivalent in $R[x]$. Similarly, Gilmer and Heinzer [7] proved that a power series ring $R[[x]]$ is Laskerian if and only if $R$ is Noetherian (but $R$ need not be Noetherian if $R[[x]]$ is a ZD-ring). We remark that Radu [13] also gave a very simple proof to show that if $R[x]$ (resp. $R[[x]]$ ) is Laskerian, then $R$ is Noetherian. His proof uses the fact that Laskerian rings satisfy (accr), so it also proves the next Theorem 2. It is worthwhile to introduce the proof here.

Theorem 2. $R[x]$ (resp. $R[[x]]$ ) satisfies (accr) if and only if $R$ is Noetherian. Proof. It is enough to prove the "only if" part of the theorem. Assume, to the contrary, that $R[x]$ satisfies (accr) and $R$ is not Noetherian; let $I_{0} \subseteq I_{1} \subseteq I_{2} \subseteq$ $\cdots$ be an ascending chain of ideals of $R$. Let $J$ be the ideal of $R[x]$ consisting of all polynomials of the form $f=\sum_{i=0}^{n} a_{i} x^{i}$, where $a_{i} \in I_{i}$ for every $i=$ $0,1, \ldots, n$ and $n \in Z^{+}$. Then we obtain the following nonterminal ascending chain of ideals of $R[x]: J:(x) \subseteq J:\left(x^{2}\right) \subseteq J:\left(x^{3}\right) \subseteq \cdots \subseteq J:\left(x^{i}\right) \subseteq \cdots$. In fact, if $a \in I_{i+1}-I_{i}$, then $a x^{i+1} \in J$ but $a x^{i} \notin J$. Thus $a \in J:\left(x^{i+1}\right)$ but $a \notin J:\left(x^{i}\right)$; therefore, $J:\left(x^{i}\right) \varsubsetneqq J:\left(x^{i+1}\right)$ for every $i \in Z^{+}$. In an analogous way, we can prove the theorem for $R[[x]]$ (cf. [13, Proof of Theorem 8.1, p. 79]).

\section{ARithmetical Rings SATISFYing (ACCR)}

A valuation ring (or a generalized valuation ring) is a ring $R$ that satisfies one of the following three equivalent conditions:

(i) for any two elements $a$ and $b$, either $a$ divides $b$ or $b$ divides $a$;

(ii) the ideals of $R$ are linearly ordered by inclusion;

(iii) $R$ is a quasi-local ring and every finitely generated ideal is principal.

We shall denote a valuation ring $R$ with the maximal ideal $m$ by $(R, m)$.

Theorem 3. A valuation ring $(R, m)$ satisfies (accr) if and only if $\bigcap_{n=1}^{\infty}\left(b^{n}\right)=(0)$ for every $b \in m$.

Proof. The necessity follows from [11, Theorem 3, p. 309]. Conversely, let $\left\{A: b^{k}\right\}_{k \in Z^{+}}$be an ascending chain of ideals, where $A$ is an ideal of $R$ and $0 \neq b \in m$. Firstly, we consider the case that $A \neq(0)$. If $b \in A$, then clearly $A: b^{k}=R$ for every $k \in Z^{+}$. On the other hand, if $b \notin A$ then $A \varsubsetneqq(b)$. Since $A \neq(0)$ and $\bigcap_{n=1}^{\infty}\left(b^{n}\right)=(0)$, there must exist a positive integer $\lambda$ such that $A \subseteq\left(b^{\lambda}\right)$ but $A \nsubseteq\left(b^{\lambda+1}\right)$. Consequently, $\left(b^{\lambda+1}\right) \varsubsetneqq A$ and therefore $A: b^{\lambda+1}=A: b^{\lambda+j}=R$ for every $j \in Z^{+}$. Secondly, we 
assume that $A=(0)$ for the chain of ideals $\left\{A: b^{k}\right\}_{k \in Z^{+}}$. If $(0): b^{k}=(0)$ for every $k$, then obviously the chain of ideals terminates. Otherwise, there exists an $i \in Z^{+}$such that $(0): b^{i} \neq(0)$. If we put $(0): b^{i}=B$ then $\left\{(0): b^{k}\right\}_{k=i+1}^{\infty}=\left\{B: b^{k}\right\}_{k \in Z^{+}}$terminates by the above case $A \neq(0)$. Hence the chain of ideals $\left\{A: b^{k}\right\}_{k \in Z^{+}}$terminates in all cases.

Corollary 1. Let $(R, m)$ be a valuation ring that is not an integral domain. Then the following statements are equivalent:

(1) $R$ satisfies (accr);

(2) every element in $m$ is nilpotent;

(3) $\operatorname{dim} R=0$;

(4) $R$ is a Laskerian ring.

Proof. Assume (1) and let $0 \neq a \in m$. According to [1, Theorem 3.1, p. 19], exactly one of the following occurs for the ideal $(a)$ : (i) $(a)=\left(a^{2}\right)$ is prime; (ii) $\left(a^{n+1}\right) \varsubsetneqq\left(a^{n}\right)$ for all $n$ and $\bigcap_{n=1}^{\infty}\left(a^{n}\right)$ is a prime ideal; (iii) $a^{n}=0$ for some positive integer $n$. Since $\bigcap_{n=1}^{\infty}\left(a^{n}\right)=(0)$ for $a \neq 0$ by Theorem 3 and $R$ is not an integral domain, (iii) is the only possible case; hence $(1) \Rightarrow(2)$. (2) $\Rightarrow$ (3) follows from the fact that $m=\operatorname{nil}(R)$, the nilradical of $R .(3) \Rightarrow(4)$ is easy to see and $(4) \Rightarrow(1)$ is due to [11, Proposition 3, p. 307].

Corollary 2. Let $(V, m)$ be a valuation domain that is not equal to its quotient field. Then the following statements are equivalent:

(1) $V$ is completely integrally closed;

(2) $V$ has rank one;

(3) $V$ is a Laskerian ring,

(4) $\bigcap_{n=1}^{\infty}\left(b^{n}\right)=(0)$ for every $b \in m$;

(5) $V$ satisfies (accr).

Proof. The equivalence of (1), (2), and (3) is a combined result of [10, Theorem 5.19 , p. $113 ; 6$, Exercise 7, p. 456]. We have seen the equivalence of (4) and (5) in Theorem 3. (2) implies (5) by [11, Theorem 6, p. 313]. To show that (5) implies (2), let $p$ be a prime ideal properly contained in $m$ and $b \in m-p$. Then $p \subseteq\left(b^{n}\right)$ for each positive integer $n$ so that $p \subseteq \bigcap_{n=1}^{\infty}\left(b^{n}\right)$. Since $\bigcap_{n=1}^{\infty}\left(b^{n}\right)=(0)$ by Theorem $3, p=(0)$ and $m$ is the only nonzero prime ideal of $V$. Hence, $V$ has rank one. So (5) implies (2) and this completes the proof.

A ring $R$ is called an arithmetical ring if its lattice of ideals is distributive, i.e., $A \cap(B+C)=A \cap B+A \cap C$ for all ideals $A, B$, and $C$ of $R$. It is well known [10, Exercise 18, p. 150] that $R$ is an arithmetical ring if and only if for each maximal ideal $m$ of $R$ the ideals of $R_{m}$ are linearly ordererd, i.e., $R_{m}$ is a valuation ring. Thus applying Corollaries 1 and 2, Theorem 3 and [11, Proposition 7, p. 308], we can obtain

Corollary 3. (1) If an arithmetical ring $R$ satisfies (accr) then $R$ has dimension at most one. (2) A Prüfer domain $D$ satisfies (accr) if and only if $D$ has dimension at most one.

We remark that, unlike the case of valuation domains, a Prüfer domain having dimension at most one is not necessarily a Laskerian ring [6, Exercise 9, p. 456].

An almost Dedekind domain is an integral domain $D$ such that $D_{m}$ is a Noetherian valuation domain for every maximal ideal $m$. Clearly, it is a Prüfer domain having dimension at most one. 
Corollary 4. Every almost Dedekind domain satisfies (accr).

In [11] it was shown that if a ring (resp. module) satisfying (accr) is equipped with an ideal-adic topology, then it satisfies some properties of a Noetherian topological ring (resp. module). In particular, if a coherent ring $R$ satisfies (accr) and is equipped with a $B$-adic topology, where $B$ is a finitely generated ideal of $R$, then $R$ behaves like a Zariski ring. More precisely, (i) the Hausdorff completion $R^{*}$ of $R$ for the $B$-adic topology is a flat $R$-module and (ii) $R^{*}$ is a faithfully flat $R$-module if and only if $B \subseteq J(R)$, the Jacobson's radical; when this is the case, all ideals of $R$ are closed [11, Proposition 8 and Theorem 8].

We have seen in Corollary 3 to Theorem 3 that every Prüfer domain of dimension one satisfies (accr). Moreover, every Prüfer domain is coherent. Thus, Prüfer domains having dimension one are coherent rings satisfying (accr). Note that they are not necessarily Noetherian or Laskerian in view of the remark made after Corollary 3 to Theorem 3. The following corollary for Prüfer domains having dimension one follows from Proposition 8, Theorem 8 and its Corollary 1, and Corollary to Theorem 9, all of [11].

Corollary 5. Let $D$ be a Prüfer domain having dimension one, $B=\left(b_{1}, b_{2}, \ldots\right.$, $\left.b_{n}\right)$ a finitely generated ideal of $D$, and $D^{*}$ the Hausdorff completion of $D$ for the B-adic topology. Then

(1) $D^{*}$ is a flat $D$-module;

(2) $D^{*}$ is a faithfully flat $D$-module if and only if $B \subseteq J(D)$; when this is the case, (i) every ideal of $D$ is closed for the B-adic topology, and (ii) $D^{*} \cong$ $D\left[\left[x_{1}, x_{2}, \ldots, x_{n}\right]\right] /\left(x_{1}-b_{1}, x_{2}-b_{2}, \ldots, x_{n}-b_{n}\right)$;

(3) if $M$ is a finitely generated D-module, $N$ and $F$ are finitely generated submodules of $M$ and $M^{*}$ is the Hausdorff completion of $M$ for the B-adic topology, then we have $M^{*} \cong D^{*} \otimes M,(N+F)^{*}=N^{*}+F^{*},(N \cap F)^{*}=N^{*} \cap F^{*}$, and $(N: F)^{*}=N^{*}: F^{*}$, where $*$ indicates completions of submodules.

\section{Finitely GENERATED MODULES OVER RINGS SATISFYING (ACCR)}

Let $R$ be a ring satisfying (accr) and $M$ a finitely generated module over $R$. We shall prove that $M$ also satisfies (accr). The proof is similar to that of the well-known fact "a finitely generated module over a Noetherian ring is Noetherian."

Theorem 4. Let $0 \rightarrow M^{\prime} \rightarrow M \rightarrow M^{\prime \prime} \rightarrow 0$ be an exact sequence of $R$-modules. Then $M$ satisfies (accr) if and only if $M^{\prime}$ and $M^{\prime \prime}$ satisfy (accr).

Proof. Suppose $M$ satisfies (accr). By [11, Proposition 6, p. 307], $M^{\prime \prime}$ satisfies (accr), and trivially $M^{\prime}$ satisfies (accr). Conversely, suppose $L$ is a submodule of $M$ such that $L$ and $M / L$ satisfy (accr). It suffices to show that $M$ satisfies (accr). For any submodule $N$ of $M$ and any element $b$ of $R$, we consider the ascending chain $N: b \subseteq N: b^{2} \subseteq N: b^{3} \subseteq \cdots$. Since both $L$ and $M / L$ satisfy (accr) and $(N+L) / L:_{M / L} b^{\lambda}=\left((N+L):_{M} b^{\lambda}\right) / L$ for any $\lambda \in Z^{+}$, there must exist positive integers $s$ and $t$ such that $(N \cap L):_{L} b^{t}=(N \cap L):_{L} n^{t+j}$ and $(N+L):_{M} b^{s}=(N+L):_{M} b^{s+j}$ for every $j \in Z^{+}$. We claim that $N: b^{s+t}=N: b^{s+t+1}$. Clearly $N: b^{s+t} \subseteq N: b^{s+t+1}$. If $x \in N: b^{s+t+1}$ then $x b^{s+t+1} \in N \subseteq N+L$. Hence, $x \in(N+L): b^{s+t+1}=(N+L): b^{s}$, 
so that $x b^{s}=u+v$ for some $u \in N$ and $v \in L$. Consequently, $x b^{s+t+1}=$ $(u+v) b^{t+1}=u b^{t+1}+v b^{t+1} \in N$. It follows that $v b^{t+1} \in N \cap L$, whence $v b^{t} \in N \cap L$ as $v \in(N \cap L):_{L} b^{t+1}=(N \cap L):_{L} b^{t}$. Now, we have that $x b^{s+t}=\left(x b^{s}\right) b^{t}=(u+v) b^{t}=u b^{t}+v b^{t} \in N$, i.e., $x \in N: b^{s+t}$, which means that $N: b^{s+t+1} \subseteq N: b^{s+t}$. Hence $N: b^{s+t+1}=N: b^{s+t}$, and we can conclude that $M$ satisfies (accr). This completes the proof of the theorem.

Theorem 5. If a ring $R$ satisfies (accr), then every finitely generated $R$-module satisfies (accr).

Proof. By Theorem 4, a finitely generated free $R$-module satisfies (accr). Every finitely generated $R$-module is the homomorphic image of a finitely generated free $R$-module. By Theorem 4 again, every finitely generated $R$-module satisfies (accr).

Corollary 1. Let $N_{1}, N_{2}, \ldots, N_{k}$ be submodules of an $R$-module $M$. Suppose that, for each $i, M / N_{i}$ satisfies (accr). Then $M /\left(N_{1} \cap N_{2} \cap \cdots \cap N_{k}\right)$ satisfies (accr).

Corollary 2. Let $M$ be a finitely generated $R$-module and $A=\operatorname{Ann}_{R} M$. Then $M$ satisfies (accr) if and only if the quotient ring $R / A$ satisfies (accr).

Proof. Refer to the proof of Theorem 2 of [12, p. 180].

\section{REFERENCES}

1. D. D. Anderson, J. Matijevic, and W. Nichols, The Krull intersection Theorem. II, Pacific J. Math. 66 (1976), 15-22.

2. M. F. Atiyah and I. G. MacDonald, Introduction to commutative algebra, Addison-Wesley, Reading, MA, 1969.

3. J. A. Beachy and W. D. Weakley, Piecewise Noetherian rings, Comm. Algebra 12 (1984), 2679-2706.

4. N. Bourbaki, Algèbre commutative, Chapters 1 and 2, Hermann, Paris, 1961.

5. __ Algèbre commutative, Chapters 3 and 4, Hermann, Paris, 1961.

6. R. Gilmer, Multiplicative ideal theory, Marcel Dekker, New York, 1972.

7. R. Gilmer and W. Heinzer, The Laskerian property, power series rings and Noetherian spectra, Proc. Amer. Math. Soc. 79 (1980), 13-16.

8. W. Heinzer and D. Lantz, N-rings and ACC on colon ideals, J. Pure Appl. Algebra 32 (1984), 115-127.

9. W. Heinzer and J. Ohm, On the Noetherian-like rings of E. G. Evans, Proc. Amer. Math. Soc. 34 (1972), 73-74.

10. M. Larsen and P. J. McCarthy, Multiplicative theory of ideals, Academic Press, New York, 1971.

11. C. P. Lu, Modules satisfying ACC on a certain type of colons, Pacific J. Math. 131 (1988), 303-318.

12. D. G. Northcott, Lessons on rings, modules and multiplicities, Cambridge Univ. Press, 1968.

13. N. Radu, Descompunerea primară in inele comutative, Lectii de Algebră III, Universitatea din Bucuresti, 1981.

Department of Mathematics, University of Colorado, Denver, Colorado 80217-3364 